\title{
Modelos de desarrollo y cambio climático
}

\author{
Blanca Azcárate y Alfredo Mingorance
}

\begin{abstract}
RESUMEN ABSTRACT
En la comunidad internacional existe unanimidad sobre la crisis ambiental planetaria. El grado de apreciación de

la misma está ligado al nivel de desarrollo, y la conciencia de participación en ella es mayor en los países ricos. Sin embargo, la gobabilidad de esta crisis implica tanto al mundo desarrollado como al menos desarrollado. El cambio climático es un claro ejemplo de la magnitud de los problemas medioambientales. Ahora bien, la aplicación de políticas preventivas y medidas correctoras evidencian la dificultad para conjugar intereses muy diversos.

There is unanimity in the international community on the subject of the earth's environmental crisis. The degree of appreciation of this problem is linked to the level of development and the participative conscience in it is greater in the rich countries. However, the global aspect of this crisis implies both the developed world and the less developed world. Climate change is a clear example of the size of environmental problems. But the application of preventive policies and corrective measures show the difficulties to combine very different interests.
\end{abstract}

\section{INTRODUCCIÓN}

El ideal de progreso ha sido una constante en la historia de la civilización. El crecimiento indefinido se fundamenta en el aprovechamiento de los recursos naturales, legítima aspiración de satisfacer las necesidades económicas y sociales de la sociedad en un medio natural. Esta incesante actividad humana en busca del desarrollo no ha valorado suficientemente el grado de deterioro de un medio ambiente vulnerable. La propia 
consideración de "recurso natural», bien de la naturaleza con reconocimiento económico, implica una dimensión finita de agotabilidad, que el hombre debe respetar.

En la sociedad preindustrial el escaso avance tecnológico no permitía una explotación desmesurada de los recursos energéticos y no energéticos. No se percibian ritmos de cambio, y la estabilidad resultaba ser la tónica dominante en una sociedad caracterizada por un consumo agropecuario. Junto a las limitaciones impuestas por la escasez tecnológica, habrá que esperar varios siglos para ver como la ciencia auxilia a la tecnología. El hombre medieval todavía vive inmerso en patrones de autoconsumo y se sirve de recursos renovables como el agua, el viento y el sol, y no consigue abrirse camino en un horizonte demasiado inabordable para su época. Todo cambia bruscamente con las transformaciones tecnológicas que tienen lugar con la Revolución Industrial. A mediados del siglo XIX, se empieza a percibir la idea de recurso asociada a la de producción: el carbón, el hierro y el acero se convierten en exponentes de consumo para una sociedad que inicia el despegue económico y que aún no es consciente de la mejora de la calidad de vida.

La sociedad moderna basa su desarrollo energético en la disminución del consumo por las mejoras en la eficiencia eléctrica (Ruesga/Durán, 1995). Hasta la utilización de los combustibles fósiles se ha aprovechado el bosque como recurso energético. El bosque amenazado por la deforestación dará paso al aprovechamiento de las energías fósiles, carbón y posteriormente petróleo, para culminar el proceso energético con la energía hidroeléctrica, la energía nuclear y más tarde con las energías renovables.

Este uso de combustibles sólidos, líquidos y gaseosos en el transporte, en las aplicaciones domésticas y en la generación de energía termoeléctrica ha contribuido de modo significativo a incrementar los índices de contaminación local y regional, y provocar el efecto invernadero a escala planetaria.

Las crisis energéticas en el mercado del petróleo, en las décadas setenta y ochenta, fueron las primeras llamadas de atención a una sociedad del derroche a la que se le exige un cambio de estrategia en la utilización de las fuentes de energía. Las perspectivas que se vaticinan de energia fósiles y/o de energías alternativas, aconsejan el ahorro y eficiencia energética. Así, el futuro energético está ligado a tres grupos de países con distinto orden de prioridades:

a) Los países desarrollados, donde se tiende cada vez más a la aplicación de medidas basadas en programas de fomento del ahorro y diver- 
sificación energética para alcanzar una mayor eficiencia, como es el caso, por ejemplo, de los estados miembros de la UE: el sistema de calefacción y aislamiento térmico (Alemania), el apoyo y promoción de energías renovables (Austria), o la regulación de normativas que promuevan proyectos de I+D.

b) El grupo de paises en desarrollo de Asia y Latinoamérica, con un fuerte crecimiento del consumo energético, tendencia que continuará en las próximas décadas.

Los dos colosos demográficos, China e India, aumentarán su consumo paralelamente a su previsible desarrollo industrial, basado en la demanda de combustibles comerciales y en el suministro energético. Los futuros mayores consumidores de energía se convertirán, así, en los mayores emisores de $\mathrm{CO}_{2}$, indisociablemente unido a las energías fósiles.

c) Los países con economía de transición o ex-países socialistas, preocupados cada vez más por un uso más racional de la energía y la reducción de la contaminación local y regional. Los países de la Europa del Este, en fase de reconversión económica, para converger con los países occidentales se verán obligados a corregir sus políticas energéticas e industriales y aceptar aquellos imperativos legales exigidos por la globalidad de la problemática ambiental.

El reto más importante consistirá en reducir el consumo per cápita, mejorando la eficiencia en los rendimientos y en la producción, si bien las energías fósiles continuarán siendo las principales suministradoras, sobre todo el gas natural, lo que supondrá un incremento de las emisiones de metano. Las predicciones de la Agencia Internacional de Energía (AIE) y el Consejo Mundial de Energía, recuerdan la tendencia creciente del consumo de energías fósiles hasta noventa millones de barriles petróleo/día en los próximos años (Soler, M., 1997).

\section{CONTAMINACIÓN AMBIENTAL Y DEGRADACIÓN DEL MEDIO}

El carácter globalizador de la problemática del deterioro ambiental es patente. Sin embargo, ésta es percibida de forma diferente según el nivel de desarrollo de las distintas regiones del globo:

a) Los países ricos, más desarrollados, donde se ha alcanzado un estado de bienestar debido a la implantación de un modelo de desarrollo que ha sido agresivo con el medio, la conciencia de degradación es mayor. Es decir, máximo desarrollo, incremento de la contaminación ambiental y una mayor sensibilidad ecológica. 
b) Los paises pobres, menos desarrollados, donde las preocupaciones comienzan en asegurar las propias necesidades básicas, el problema del deterioro ambiental pasa a un segundo plano.

No resulta fácil conjugar intereses tan contrapuestos entre la riqueza del norte y la pobreza del sur. Cuando se aborda la contaminación ambiental y la degradación del medio, a pesar de los diferentes intereses en juego, existe unanimidad en la percepción del problema. Las discrepancias surgen en las posturas a adoptar para corregir o minimizar sus consecuencias, es decir, hay un distinto grado de valoración. Sin embargo, hay que asumir el compromiso y aceptar las responsabilidades políticas derivadas de un mayor o menor grado de desarrollo.

En la reciente Conferencia de Kyoto (1997) se ha puesto de relieve esta controversia entre los países con diferente grado de desarrollo, así como también han surgido distintas interpretaciones dentro de los países ricos: la Unión Europea, por un lado y Estados Unidos y Japón, por otro. Hasta ahora tan sólo se ha conseguido un compromiso testimonial e irrelevante sobre un peligro futuro, ya una amenaza presente: la contaminación ambiental. (Álvarez de Miranda, 1995).

Para alcanzar un medio ambiente mejor, es posible optar por un cambio del modelo de consumo o por distintos mecanismos de producción, ambas opciones basadas en el empleo de tecnología verde. El crecimiento económico debe y puede preservar y restaurar la naturaleza, según Mas-Colell «es por nuestra riqueza, que no por nuestra pobreza, como salvaremos la naturaleza” (Ballesteros y Pérez Adán, 1997).

No es válido, por frágil y débil, el crecimiento apoyado en el agotamiento de los recursos no renovables en un medio deteriorado por los residuos industriales, la contaminación, el cambio climático-efecto invernadero, la destrucción de la capa de ozono, etc... Los procesos antrópicos en el medio natural se han caracterizado hasta ahora por la sobreexplotación de los recursos, los vertidos sin control y la proliferación de residuos, sobre todo industriales, no reciclables en la biosfera. Los efectos de estas actuaciones se manifiestan en la contaminación global, en los problemas macroecológicos del cambio climático y el efecto invernadero, en el agujero de la capa de ozono, en la pérdida de biodiversidad; y en los problemas microecológicos, en la contaminación local del aire por las emisiones de monóxido de carbono, óxido de nitrógeno, óxido de azufre, compuestos orgánicos volátiles (COV) y partículas en suspensión (Arroyo et al., 1997).

El cambio climático ha sido, entre los problemas macroecológicos, el que ha despertado una mayor sensibilidad en la sociedad. Cambio climá- 
tico o variabilidad del clima puede deberse a fluctuaciones naturales o causas antropogénicas que permiten el establecimiento de predicciones. Se considera necesario un periodo de treinta años para caracterizar los rasgos climáticos de una región o área espacial, y analizar la variabilidad atmosférica. Esta diversidad y variabilidad climática es la respuesta a los balances de energía Tierra/Atmósfera y por tanto de la radiación solar. La variación de la constante solar o irradiancia (radiación solar/superficie terrestre) significará aumento o disminución de la temperatura del globo o efecto invernadero.

El efecto invernadero es el balance de energía Tierra/Atmósfera modificado por la radiación terrestre o radiación térmica infrarroja. Si los gases de invernadero, vapor de agua, $\mathrm{CO}_{2}$, Nox, metano, ozono y CFCs aumentan su concentración en la atmósfera pueden modificar el balance energético del sistema Tierra/Atmósfera e influir en un posible cambio climático planetario.

El principal gas invernadero, el vapor de agua, está presente en la atmósfera sin posibilidad de aumentar su concentración. Procede de la evaporación de los océanos y de los mares, cambia fácilmente de estado, se condensa en forma de vapor y precipita cuando aumenta excesivamente la concentración, es decir, alcanza el estado de saturación.

El $\mathrm{CO}_{2}$ es uno de los gases de mayor presencia. Su concentración ha aumentado de manera significativa en la segunda mitad de este siglo: a comienzos de siglo se contabilizaron $290 \mathrm{ppm}$ (partes por millón de volumen) y a finales más de $355 \mathrm{ppm}$. Si las emisiones de $\mathrm{CO}_{2}$ se mantuvieran al ritmo actual, a finales del próximo siglo, las concentraciones alcanzarían valores aproximados de $500 \mathrm{ppm}$. Este inquietante aumento se debe principalmente a la combustión de energías fósiles (carbón, petróleo y sus derivados), al transporte (motores de explosión) y a las centrales termoeléctricas como grandes emisoras de $\mathrm{CO}_{2}$, que la biosfera es incapaz de absorber, a pesar de los sumideros del ciclo del carbono (océanos y fotosíntesis de las masas boscosas). El aire atmosférico se carga de $\mathrm{CO}_{2}$, las concentraciones aumentan a un ritmo de $0,5 \%$ anual y originan un aumento térmico en las capas bajas atmosféricas (Lorente, 1997). Este aumento queda reflejado en las emisiones anuales per cápita: 
Emisiones de $\mathrm{CO}_{2}(1995)$ por persona y año

\begin{tabular}{ll}
\hline ÁREA & TN/AÑO \\
\hline Estados Unidos & 19,88 \\
Canadá & 15,90 \\
Australia & 15,84 \\
Arabia Saudí & 11,96 \\
Holanda & 11,57 \\
Alemania & 11,18 \\
Rusia & 10,83 \\
Reino Unido & 9,64 \\
Japón & 9,17 \\
Corea del Sur & 7,87 \\
Sudáfrica & 7,74 \\
Italia & 7,40 \\
España & 6,30 \\
Francia & 6,23 \\
México & 3,46 \\
\hline
\end{tabular}

Fuente: Agencia Internacional de la Energía.

No sólo el $\mathrm{CO}_{2}$ absorbe la radiación infrarroja terrestre, otros gases participan también en la absorción, como es el caso del Nox, metano, ozono y CFCs, pero en concentraciones todavía bastante bajas. De ahi que se expresen en ppb (partes por billón de volumen), aunque pueden aumentar significativamente.

Si por el efecto invernadero la troposfera se calienta y las altas capas de la atmósfera se enfrían, el balance global de energía a escala planetaria se altera en este proceso. La energía solar permanece invariable, pero la radiación terrestre resulta modificada. Se trata de un calentamiento adicional que puede explicarse por el efecto invernadero natural, debido a la presencia de gases (vapor de agua y $\mathrm{CO}_{2}$ principalmente) en la atmósfera, que absorben parcialmente la radiación emitida por la Tierra y la devuelven a la superficie terrestre. Esto explica que la radiación saliente se equilibre con la radiación solar entrante y exista un calentamiento natural vital necesario para la habitabilidad de la Tierra. El problema surge cuando se incrementa bruscamente ese efecto invernadero que provoca un rápido calentamiento en la superficie terrestre, debido a las actividades humanas que aumentan las concentraciones atmosféricas de los gases de efecto invernadero (Bautista Pérez, 1995).

Al analizar los ciclos glaciales e interglaciales observamos variaciones de temperatura en la superficie de la Tierra entre $5^{\circ}$ y $7^{\circ} \mathrm{C}$, y en algunas 
regiones entre $10^{\circ}$ y $15^{\circ} \mathrm{C}$ (Bautista Pérez, 1995). Desde la última glaciación, aproximadamente hace 10.000 años, las temperaturas de la superficie terrestre han fluctuado poco más de $1^{\circ} \mathrm{C}$, mientras que en el último siglo el aumento térmico ha sido entre $0,3^{\circ}$ y $0,6^{\circ} \mathrm{C}$, y en las últimas décadas se han registrado aumentos de entre $0,2^{\circ}$ y $0,3^{\circ} \mathrm{C}$.

Es previsible, pues, un progresivo calentamiento de la superficie del globo, si se mantienen elevados niveles de $\mathrm{CO}_{2}$ y se duplican las concentraciones actuales. El calentamiento global supondrá una modificación del balance energético y un cambio en la circulación general de la atmósfera, distinta localización del frente polar, las masas de aire presentarán nuevos contrastes térmicos, mayores totales pluviométricos por aumento de la evaporación y concentración de vapor de agua. Además hay que tener en cuenta los factores locales o regionales (altitud, orografía, distancia del mar, etc.) para explicar la nueva situación. A grandes rasgos, se pueden establecer una serie de consecuencias, entre las que se pueden citar:

- La fusión de las masas de hielo. El comienzo de la fusión parcial de los casquetes polares de gran poder reflectante de la radiación solar (alto albedo).

- Modificación del régimen pluviométrico, (con la consiguiente repercusión en las inundaciones y sequias). Asi, en algunos casos, aumentarían de una manera generalizada las precipitaciones y la intensidad (cantidad/superficie/tiempo $=$ litros $/ \mathrm{m}^{2} /$ hora) de las mismas.

- Ascenso del nivel del mar, lo que provocará una devastación de las áreas costeras. El nivel del mar ha subido entre 10 y $25 \mathrm{~cm}$. a lo largo de este siglo.

La subida del nivel del agua en mares y océanos, originaría un riesgo de inundación en países como Bangla Desh y Holanda, con bajas zonas costeras. Las observaciones a lo largo de este siglo indican un pequeño aumento, pero progresivo, del nivel del agua oceánica que puede deberse a la dilatación térmica del agua, los vientos, las corrientes marinas y, tal vez, a movimientos de subsidencia debidos a procesos de sedimentación - a placas tectónicas en movimiento, con valores que oscilan entre 0,5 y 3 $\mathrm{mm} / a n ̃ o$. Consecuencias derivadas de esta subida del nivel del agua son el aumento de la salinidad en aguas superficiales y el ascenso del nivel freático en las costas (Lorente, 1997).

- Incremento de las áreas desérticas.

- Extinción de especies de flora y fauna. 
- Incremento de enfermedades tropicales, disminución del número de cosechas... etc.

Desde el punto de vista científico cabe plantearse una serie de incertidumbres sobre el comportamiento del sistema climático planetario:

- Comportamiento de los gases de invernadero en una atmósfera recalentada.

- El papel de los sumideros, principalmente de los océanos.

- La influencia de la nubosidad y las masas de hielo de los polos (criosfera).

Para dar respuesta a estas incógnitas se emplean modelos climáticos de previsión y simulación, por ahora de escasa fiabilidad, debido a la complejidad de las técnicas de modelización. Resulta complicado conocer con exactitud los valores de las variables observadas y conjugarlos con valores reales, si se opta por simplificar no es posible conocer fiablemente el estado de la atmósfera. Es por ello, que las predicciones meteorológicas carecen de fiabilidad a partir de un plazo de una semana, si tenemos en cuenta el imprevisible comportamiento de la circulación general de la atmósfera.

En las proyecciones realizadas para el próximo siglo se recogen distintos escenarios con diferentes concentraciones de emisiones de $\mathrm{CO}_{2}$, es decir, con una variedad tipológica de situaciones:

- Emisiones de $\mathrm{CO}_{2}$ al ritmo actual, que reflejará una mayor deforestación de los bosques tropicales.

- Mayor consumo de combustibles con menor contenido en carbono, como es el caso del gas natural, unido a la mejora de la eficiencia energética llevará consigo la estabilización de las emisiones de CO2 en los valores actuales.

- Mayor aprovechamiento de las energías renovables y de la energía nuclear en el futuro y la sustitución de los CFCs, significará un importante cambio cualitativo, al disminuir las emisiones.

- La reducción de las emisiones de gases de invernadero (hasta un $50 \%$ ) en los países desarrollados y la previsión de un ligero aumento en los países en desarrollo, junto a una importante contribución de las energías no emisoras de $\mathrm{CO}_{2}$ supondrá una nueva situación (Soler, 1997).

A pesar de los diferentes escenarios previstos por las predicciones, la mayoría de los expertos coinciden en que el efecto invernadero se está intensificando por causas antropogénicas, y que el calentamiento del plane- 
ta dará lugar a cambios a medio y largo plazo, aunque las dudas surgen al valorar la magnitud y el ritmo del calentamiento.

Ante la previsión de un cambio climático cabe desarrollar una serie de estrategias para aplicar a corto y a largo plazo (Bautista Pérez, 1995):

\section{A corto plazo:}

- La mejora de la eficiencia energética reduce las emisiones de $\mathrm{CO}_{2}$.

- Tecnologías y fuentes más limpias disminuyen las emisiones de $\mathrm{CO}_{2}$.

- La reforestación supone mayor absorción de los sumideros de $\mathrm{CO}_{2}$.

- Sustitución de los CFCs y protección del ozono estratosférico.

- Ordenación y protección de zonas costeras inundables.
A largo plazo:

- Desarrollo de programas de I+D.

- Aplicación de tecnologías verdes o ecologías en la energía e industria.

- Cambio de hábitos sociales: transporte en zonas urbanas, mejorar la eficiencia de los recursos y el reciclado de los residuos.

Por último, para entender el cambio climático y las estrategias a desarrollar se deben tener en cuenta una serie de consideraciones:

- El cambio climático es una amenaza, pendiente de confirmación científica, que se cierne sobre el planeta.

- El compromiso debe ser asumido por todos los países, por la globalidad de sus efectos.

- Las políticas ambientales, necesariamente deben contemplar un nuevo modelo energético que minimice las emisiones de $\mathrm{CO}_{2}$, al tiempo que disminuya progresivamente el consumo de energías fósiles.

- Los países desarrollados están moralmente obligados a facilitar tecnología y transferir disponibilidades a los países en desarrollo para afrontar un proyecto de cambio común.

- La diversidad de intereses económicos no debe prevalecer sobre un objetivo único: la protección ambiental de la biosfera. 
Otro tipo de problemas se presentan asociados a los contaminantes secundarios derivados de la combustión de energías fósiles y la biomasa, como es el caso del dióxido de nitrógeno, el ácido nítrico, el ácido sulfúrico y los sulfatos y nitratos que precipitan en forma de lluvia ácida al entrar en contacto con la humedad atmosférica. Si la precipitación es seca se forman gases, nieblas y partículas en suspensión. Este problema de la lluvia ácida, también traspasa los límites regionales y se convierte en transfronterizo: por ejemplo, las áreas industrializadas de la Europa del Este, son un foco emisor de contaminantes secundarios, donde el viento, agente de transporte, los convierte en un grave problema en los países vecinos como Noruega, Finlandia, Suecia, Alemania y Austria.

Un medio contaminado, por procesos variados, es el agua: residuos domésticos e industriales, escorrentía en tierras cultivadas tratadas con herbicidas y pesticidas o los estériles de las cuencas mineras. La situación se agrava en las zonas eutrofizadas o aguas estancadas, donde se acumulan fosfatos y nitratos y materias orgánicas, que reducen de manera drástica las especies al proliferar las condiciones anaerobias en estos ecosistemas.

En las grandes urbes otro grave y difícil problema de abordar es el tratamiento de los residuos sólidos urbanos (RSU) y su eliminación. La controvertida incineración es una opción que cuenta con la ventaja de reducir el volumen y generar energía, aunque no exenta de inconvenientes al liberar a la atmósfera contaminantes gaseosos, cenizas y partículas tóxicas en suspensión como el plomo, mercurio y cadmio. No hay que transferir la contaminación de un medio a otro. Los problemas medioambientales exigen soluciones y la tecnología ayuda a corregirlos y no a solucionarlos.

La sociedad de consumo de masas ha significado el comienzo del deterioro medioambiental y para atajarlo es necesario definir lo que se considera impacto ambiental o destrucción de hábitats, pérdida de recursos florísticos y faunísticos, pérdida de masa forestal, desaparición de suelo agropecuario, modificación de cursos fluviales y deterioro de la red subterránea de aguas. En conjunto, alteración y degradación del paisaje (Ruesga/Durán, 1995).

\section{CRISIS ECOLÓGICA, MOVIMIENTO MEDIOAMBIENTAL Y NUEVAS ESTRATEGIAS DE DESARROLLO}

La crisis ecológica es simplemente una crisis social del modelo de producción y consumo, cuyas consecuencias más graves son la destrucción 
de recursos no renovables y la difícil eliminación de residuos no reciclables. A pesar de la diversidad de intereses en la preocupación medioambiental existen una serie de fechas históricas donde se han recogido propuestas e informes que nos acercan paulatinamente a valorar, en mayor 0 menor medida, el avance hacia el desarrollo equilibrado.

La Conferencia de Estocolmo (1972), supone la primera llamada de atención a la comunidad internacional sobre la problemática medioambiental "por ser de alcance regional o mundial, o por repercutir en el ámbito internacional común, requerirá una amplia colaboración entre las naciones y la adopción de medidas por las organizaciones internacionales en interés de todos" (Campins Eritja, 1997: 345).

En 1984/87, la Comisión Mundial sobre el Medio Ambiente y el Desarrollo, presenta el Informe Brundtland, donde se destaca la importancia de integrar desarrollo y Medio Ambiente. En 1991 se presenta el Documento «Cuidar la Tierra. Una estrategia para vivir de manera sostenible», publicado por la Unión Internacional para la Conservación de la Naturaleza (UICN), el Programa de las Naciones Unidas para el Medio Ambiente (PNUMA) y el Fondo Mundial para la Naturaleza (WWF). Este documento insiste en la necesidad de aprovechar los recursos naturales bajo el principio de la sostenibilidad, limitación del aprovechamiento de los mismos potenciando estilos de vida y modelos de desarrollo respetuosos con el medio apoyados en tecnología ambiental. Actualmente, si los países del Tercer Mundo pudieran llevar a cabo los modelos de vida y de consumo del mundo desarrollado, probablemente la capacidad de carga del planeta se vería saturada.

Toda esta preocupación se manifiesta también en la Conferencia de Río (1992), donde por primera vez se plantea la necesidad de aplicar políticas medioambientales. La Unión Europea recoge la propuesta y pone en marcha el $V$ Programa Comunitario sobre medio ambiente y desarrollo sostenible.

Uno de los organismos internacionales que han tratado de sensibilizar a la opinión pública sobre el aumento de las concentraciones de $\mathrm{CO}_{2}$ y otros gases de efecto invernadero es el llamado Panel intergubernamental del Cambio Climático (IPCC), creado en 1988 a iniciativa de la Organización Meteorológica Mundial y el Organismo de las Naciones Unidas para el Medio Ambiente. Ya en 1992 establece cinco importantes conclusiones:

- La temperatura media en la superficie terrestre ha aumentado en los últimos 100 años entre 0,3 y $0,6^{\circ} \mathrm{C}$. 
- Estas cifras se ajustan a las previsiones establecidas por los modelos climáticos, y coinciden con la variabilidad natural del clima.

- Con los datos obtenidos de los modelos de simulación y si se verifica la duplicación de las concentraciones actuales de $\mathrm{CO}_{2}$, previsiblemente la temperatura media terrestre aumentará entre 1,5 y $4,5^{\circ} \mathrm{C}$.

- Las proyecciones y predicciones establecidas sobre el cambio climático plantean aún numerosas dudas a distintas escalas, magnitud del fenómeno y distribución por áreas geográficas.

- La constatación del aumento del efecto invernadero se llevará a cabo en las próximas décadas.

Estas conclusiones formuladas por el IPCC, son claramente coincidentes con otros informes presentados recientemente como el de la primera Conferencia Intergubernamental sobre Educación Ambiental, convocada por la UNESCO en el año 1997 en Tbilisi (Georgia).

En la Conferencia de Kyoto (1997) se ha acordado reducir, para los años 2008 y 2010, las emisiones de gases que producen efecto invernadero en los países más desarrollados, una media del $5,2 \%$. Tomando como nivel de referencia el año 1990, para el $\mathrm{CO}_{2}$, y el año 1995, para el caso de los hidrofluorucarbonos, los perfluorocarbonos y el hexaflururo de carbono.

Así, dentro de los países más desarrollados, la Unión Europea se comprometió a reducir estas emisiones en un $8 \%$; Norteamérica (Estados Unidos y Canadá), en un $7 \%$; Japón en un $6 \%$. Mientras que en los paises menos desarrollados no se fijó ningún límite.

Este acuerdo queda muy por debajo del nivel necesario de reducción para evitar el llamado efecto invernadero, ya que la reducción de sólo un $5 \%$ no palia de ninguna manera el aumento de emisiones que de modo previsible se registrará en el resto de los países menos desarrollados. Según el IPCC (Panel Intergubernamental sobre Cambio Climático) las reducciones por debajo del $20 \%$ no tendrán apenas repercusiones en el calentamiento del planeta a largo plazo.

La crisis ecológica se inicia con la primera Revolución Industrial, aunque el aumento de sus magnitudes tiene lugar desde la segunda mitad del siglo xx hasta alcanzar niveles de globalidad. Esta contaminación y degradación ambiental es resultado de unas formas de desarrollo que podemos denominar insostenibles, características de las civilizaciones industriales, donde no se ha prestado importancia a los daños ecológicos y sociales, ya que a pesar del gran avance científico y tecnológico se regis- 
tra paralelamente un incremento de la pobreza para una gran parte de los habitantes de la Tierra. Por lo tanto, se deben propiciar nuevos modelos de desarrollo que puedan dar respuesta adecuada a los problemas del cambio global y que tenga capacidad de asegurar un medio ambiente y social más justo (Jiménez Herrero, 1996).

Muchas veces se relaciona el concepto desarrollo con el de crecimiento económico, en el sentido de un aumento de la capacidad de producción de una economía. Pero no hay que olvidar, que un proceso económico que implique desarrollo debe ir acompañado paralelamente de otros componentes sociales, como por ejemplo:

- que el aumento de la producción no sólo se produzca en una rama económica sino en varias,

- que haya cambio en la estructura y en el peso relativo de los diferentes sectores económicos,

- y, lo más importante, que se registre una mejora del nivel de vida de la mayoría de los habitantes.

La necesidad de reducir el abismo que media entre los países ricos del norte y los pobres del sur para conseguir una sostenibilidad global es cada vez más evidente.

Así, aunque el término desarrollo sostenible comienza a utilizarse en la década de los setenta no se generaliza su uso hasta 1986, difundido en la Comisión Mundial de Medio Ambiente y Desarrollo (CNUMAD) en el denominado Informe Brundtland y que lo entiende como "aquel que satisface las necesidades de la generación presente sin comprometer la capacidad de las generaciones futuras para satisfacer sus propias necesidades" (Martí Boada, 1997: 48). Su aceptación va unida al "desencanto desarrollista" y una mayor "concienciación ambiental" de la sociedad.

Hay que tener en cuenta, sin embargo, que el concepto desarrollo sostenible consolidado ya en esta década ha ido formándose a partir de diferentes teorías que fueron apareciendo desde principios de los años setenta que llamaban la atención sobre la necesidad de tener en cuenta la problemática ambiental y social pero que entonces no fueron tenidas en cuenta ya que desde los sesenta los modelos de desarrollo van ligados a factores puramente economicistas, ignorando aquellos factores, que van a permitir una mejor calidad de vida social y ambiental. Es el caso, por ejemplo del denominado ecodesarrollo que se se gesta en 1973 por I. Sach y que señala el comienzo del planteamiento de buscar una concordancia entre desarrollo y medio ambiente. 
El ecodesarrollo responde al modelo de producción que compatibiliza los procesos ambientales con los socioeconómicos y preserva los ecosistemas terrestres a escala local y regional. Este modelo, respetuoso con los recursos naturales se inclina hacia la sostenibilidad para garantizar las disponibilidades a las futuras generaciones.

La idea de desarrollo sostenible se impone a finales de los ochenta y a lo largo de esta década por las iniciativas llevadas a cabo por la Comisión Mundial sobre Medio Ambiente y Desarrollo que reclama la participación internacional en un Plan de Acción futuro para evitar el deterioro ambiental. "Un estilo de desarrollo ambientalmente sostenible, no sólo implica una preocupación por la salud del planeta y la supervivencia colectiva, sino que también pone especial acento en el largo plazo, el derecho de las futuras generaciones y la justicia social' (Jiménez Herrero, 1996: 98).

Ya en 1980, la Unión Internacional para la Conservación de la Naturaleza (UICN), fijó el objetivo de desarrollo sostenible como la capacidad de carga que el medio ambiente es capaz de soportar sin degradarse. Años más tarde, en 1986, el Informe Brundtland presentó los objetivos del desarrollo sostenible ligados al aumento del bienestar social, sin renunciar a resultados económicos, pero que no tiene que llevar consigo el incremento en la utilización de materias primas y energía (Pardo, 1997).

De este modo en 1972 (Conferencia de las Naciones Unidas sobre el Medio Ambiente Humano. Estocolmo) comienza la andadura hacia la integración del binomio Desarrollo/Medio Ambiente, en una constante búsqueda de la compatibilidad de ambos conceptos, hasta la Conferencia de Río (1992) (Conferencia de las Naciones Unidas para el Medio Ambiente y Desarrollo). En estos veinte años ha variado sustancialmente el tratamiento de esta problemática, y la integración del aspecto ambiental en los modelos de desarrollo ha llevado consigo la necesidad de tener en cuenta otra serie de factores tanto para las regiones industrializadas como para las menos desarrolladas.

Independientemente de la controversia Norte/Sur o desarrollo y no desarrollo, el concepto de medio ambiente debe entenderse como un bien patrimonial común que hay que preservar y proteger, y responde a una única finalidad alcanzar un crecimiento en equilibrio con el marco natural.

\section{LA PREOCUPACIÓN MEDIOAMBIENTAL EN LA UNIÓN EUROPEA}

Hemos visto como la panorámica y los análisis sobre los problemas del medio ambiente han registrado en los últimos años una importante 
transformación. Con un espectacular crecimiento demográfico y el aumento de las actividades económicas estamos asistiendo a un incremento de la contaminación y un agotamiento de los recursos naturales, unido a un desequilibrio económico entre las regiones opulentas del Norte y las más pobres del Sur.

De este modo, las políticas sobre medio ambiente han de situarse dentro del marco del llamado desarrollo sostenible para poder conseguir un progreso más globalizador, un modelo de desarrollo que tenga en cuenta los problemas que puedan derivar en las generaciones futuras (Jiménez Herrero, 1996).

En lo que respecta a la trayectoria de las políticas de la Unión Europea, en 1972 se registran los primeros avances comunitarios en materia de protección ambiental. (En 1986, con la aprobación del Acta Única Europea, se incluye por primera vez el medio ambiente en las políticas comunitarias, y en el Tratado de Maastricht (1992) se convierte en una política común). Desde entonces se han elaborado cinco Programas de Acción de la Unión Europea en materia de Medio Ambiente. El último y vigente en la actualidad, que lleva por título "Hacia un desarrollo sostenible», entró en funcionamiento en el año 1993 y aporta como novedad el concepto de desarrollo sostenible, es decir, el tratamiento del medio ambiente no como un factor limitativo del desarrollo, sino como un elemento que debe ir unido a éste. (desarrollo sostenible = "satisface las necesidades del presente sin poner en peligro la capacidad de las generaciones futuras para satisfacer sus propias necesidades").

EI V Programa comunitario en materia de medio ambiente y desarrollo sostenible (1992/2000) tiene un carácter más globalizador que los anteriores, ocupándose de cinco ámbitos de gran importancia por su repercusión en el medio ambiente: industria, energía, transporte, agricultura y turismo; y siete temas específicos: cambio climático, calidad del aire, medio ambiente urbano, zonas costeras, gestión de residuos, gestión de los recursos hídricos y protección de la naturaleza.

En el seno de la UE existen criterios medioambientales dispares entre un norte y centro comunitario desarrollado y un sur menos desarrollado. Esta distinta percepción permite establecer distintas políticas medioambientales, por un lado las que implantan los países más rigurosos como Alemania, Dinamarca y Holanda; por otro, políticas más flexibles, en los países del sur, e incluso políticas con estrategias propias y un cierto grado de laxitud, como es el caso del Reino Unido. Sin embargo, están claramente diseñados los objetivos comunitarios en la política medioambiental (Comisión Europea 1997): 
a) Conservar, proteger y mejorar la calidad del medio ambiente, y conseguir una reducción de las emisiones de contaminantes a la atmósfera.

b) Garantizar un uso racional de los recursos naturales y protección de éstos.

c) Acción preventiva y aplicación del principio contaminador/pagador.

Para lograr estos objetivos la política comunitaria en temas de medio ambiente está basada en cinco principios fundamentales:

- Principio de cautela, mediante el cual se pueden aplicar medidas sin necesidad de esperar a la comprobación científica.

- Principio de acción preventiva. Adopción de medidas de prevención ante posibles daños al medio ambiente, para evitar daños irreparables y porque supone un menor coste económico.

- Principio de corrección en la fuente. Los daños al medio ambiente deben resolverse allí donde se han producido y no trasladar el problema, caso por ejemplo de los problemas de la ubicación de residuos.

- Principio de quien contamina paga, o principio de responsabilidad.

- Principio de subsidiariedad. Las acciones llevadas a cabo por la UE se restringen a las disposiciones que requieran la intervención/mediación comunitaria.

Estos principios de aceptación general, no tienen el mismo grado de cumplimiento entre unos y otros países. Así, en España, como ejemplo de área meridional europea, todavía no se ha alcanzado un grado de sensibilidad ante las cuestiones medioambientales comparable a otros paises europeos. La falta de una política comprometida con los temas del medio ambiente explica el porqué no se adoptan medidas correctivas y preventivas. Quizás tenga justificación por el traspaso de transferencias en la gestión medioambiental a las Comunidades Autónomas, sin haber fijado previamente un criterio homogéneo.

Si tenemos que caracterizar las actuaciones administrativas, la ineficacia y la falta de coordinación son normas generalizadas en las tres administraciones (nacional, autonómica y local) y no se ha implicado a otros actores de tanta importancia en el sector privado como las instituciones empresariales y los grupos ecologistas.

No ocurre asi en Alemania, como ejemplo de país centroeuropeo con mayor concienciación ambiental, donde ya a finales de los sesenta comienza una preocupación por estos temas en la clase política, por encima 
de intereses partidistas a la que se une el movimiento ecologista. En este país, la prevención, la cooperación y la contaminación pago son principios rectores en todas las actuaciones ambientales. No hay que olvidar que en algunas zonas de este país, el alto índice de industrialización ha supuesto un alto grado de deterioro. Además se ha ido desarrollando un sector industrial verde, de gran importancia económica y con capacidad para exportar tecnología. Los Länder son los principales responsables de la legislación medioambiental.

La preocupación creciente de la sociedad por el deterioro del medio ambiente adquiere un carácter global, traspasando las fronteras nacionales y locales, y cada vez es más patente la necesidad de conservación de este medio y la promulgación de políticas correctoras adecuadas.

Por otro lado, la política ambiental de la Unión Europea se apoya en una serie de instrumentos y medidas para poder aplicar correctamente las iniciativas consideradas de interés como son, entre otras:

a) La creación de la Agencia Europea del Medio Ambiente (AEMA) en 1994 y con sede en Copenhague, con el objetivo de mejorar la información $y$ acceso a los datos sobre temas ambientales.

b) Exigencia de la realización de estudios de Evaluación de Impacto Ambiental (EIA), con antelación a la aprobación definitiva de programas o planes al desarrollo que se lleven a cabo por alguno de los Estados miembros.

c) La creación de sistemas que incentiven la protección del medio, como es el sistema de concesión de la etiqueta ecológica o el sistema comunitario de gestión y auditoría.

d) Apoyo y relevancia de los estudios de I+D en materia de medio ambiente y mayor atención a los temas relacionados con la formación, información y toma de conciencia sobre la necesidad de protección del medio ambiente.

Por último, la cooperación en el ámbito internacional es cada vez mayor. Desde 1992 engloba dentro de sus políticas los objetivos delimitados en la Declaración de Río y en el Programa 21, así como aquellos acuerdos que se fijan a nivel internacional en aspectos relacionados con el cambio climático por su carácter globalizador.

\section{BIBLIOGRAFÍA}

Álvarez de Miranda, A.: “La energía y el cambio climático». XII Jornadas Nacionales sobre Energia y Educación. Madrid, septiembre de 1995. 
Arroyo llera, F./Camarero Bullón, C. y Vazquez Varela, C. (1997): “Análisis de los problemas medioambientales". En: Ballesteros, J. y Pérez Adan, J.: Sociedad y Medio Ambiente, Madrid, págs. 49/81.

AzCARATE, B. y Mingorance, A. (1996): “La contribución de las energías renovables en la planificación energética española". Espacio Tiempo y Forma, Serie VI, 9, págs. 39-51.

Ballesteros, J. y Pérez Adán, J. (1997): Sociedad y medio ambiente, Trotta, Madrid.

Bautista PEREZ, M. (1995): "Cambio climático. Causas y posibles efectos". XII Jornadas Nacionales sobre Energía y Educación. Madrid, septiembre de 1995.

CAMPINS ERITJA, M. (1997): «La institucionalización de la cooperación internacional para la protección del medio ambiente". En: M. SOLER (coord.): Manual de gestión del medio ambiente Barcelona, págs. 339/359.

Comunidades Europeas (1993): Hacia un desarrollo sostenible. Luxemburgo.

Comisión Europea (ed.) (1997): Hacia un desarrollo sostenible. Informe de aplicación y plan de actuación de la Comisión Europea sobre el quinto programa de política y actuación en materia de medio ambiente y desarrollo sostenible. Luxemburgo.

LORENTE, J. (1997): “El cambio climático". En: M. SOLER (coord.) Manual de gestión del medio ambiente, Barcelona, págs. 118-132.

JIMÉNEZ HERRERO, L. (1996): Desarrollo sostenible y economía ecológica. Madrid.

MARTI BOADA (1997): "La educación ambiental: un instrumento para el cambio". En: M. SOLER (coord.) Manual de gestión del medio ambiente, Barcelona, págs. 44-57.

PARDO, M. (1997): «El desarrollo». En: J. Ballesteros y J. PÉrez AdAn: Sociedad y Medio Ambiente, Madrid, págs. 187/205.

Ruesga, S. y G. Duran (1995): Empresa y medio ambiente, Pirámide, Madrid.

SOLER, M. (coord.) (1997): Manual de gestión del medio ambiente. Barcelona. 